\title{
The role of nuclear EpICD in extrahepatic cholangiocarcinoma: Association with $\beta$-catenin
}

\author{
SARANGEREL JACHIN ${ }^{1}$, JUN SANG BAE ${ }^{1}$, JONG JIN SUNG ${ }^{1}$, HO SUNG PARK ${ }^{1}$, \\ KYU YUN JANG ${ }^{1}$, MYOUNG JA CHUNG ${ }^{1}$, DAE GOHN KIM ${ }^{2}$ and WOO SUNG MOON ${ }^{1}$ \\ Departments of ${ }^{1}$ Pathology and ${ }^{2}$ Internal Medicine, Chonbuk National University, Medical School, \\ Research Institute of Clinical Medicine of Chonbuk National University Hospital and \\ Research Institute for Endocrine Sciences, Jeonju 561-756, Republic of Korea
}

Received March 12, 2014; Accepted May 15, 2014

DOI: 10.3892/ijo.2014.2472

\begin{abstract}
After intramembranous proteolysis-mediated loss of the extracellular domain of the epithelial cell adhesion molecule (EpEx) and release of an intracellular domain (EpICD) into the cytoplasm, EpICD sequentially associates with FHL2 to form a nuclear complex with $\beta$-catenin and Lef-1. This association induces gene transcription involved in the activation of the oncogenic potential of epithelial cell adhesion molecule (EpCAM). We examined the localization and expression of EpEx, EpICD and $\beta$-catenin in surgical specimens of extrahepatic cholangiocarcinoma (ECC) from 79 patients and focused on the relationship between nuclear expression of EpICD and $\beta$-catenin. We also examined the role of EpICD by transfecting the EpICD cDNA in cholangiocarcinoma (CC) cell lines. There was a significant correlation between the nuclear expression of EpICD and $\beta$-catenin in ECC tissues. Frequent nuclear co-localization of EpICD and $\beta$-catenin was observed in cancer cells forming the invasive front. Nuclear expression of EpICD also significantly correlated with histologic grade of tumor. Overexpression of EpICD in the CC cells significantly increased the cell growth and proliferation. The overexpression of EpICD in the CC cells also increased the expression levels of the active form of $\beta$-catenin and EpCAM target genes, such as $c$-myc and cyclin Dl. Furthermore, the overexpression of EpICD significantly enhanced the migration and invasiveness of CC cells. Conversely, the inhibition of EpCAM in EpCAMoverexpressing cells by siRNA significantly decreased cell proliferation, migration and invasion. These results indicate that the spatial localization of EpICD and its mutual interaction with $\beta$-catenin may be important in ECC progression and invasion.
\end{abstract}

Correspondence to: Professor Woo Sung Moon, Department of Pathology, Chonbuk National University, Medical School, 567 Baekje-daero, Deokjin-gu, Jeonju-si, Jeollabuk-do 561-756, Republic of Korea

E-mail: mws@chonbuk.ac.kr

Key words: cholangiocarcinoma, epithelial cell adhesion molecule, catenin, invasion

\section{Introduction}

Epithelial cell adhesion molecule (EpCAM) is a transmembrane protein that was first proposed to function as a homophilic cell adhesion molecule that can interfere with cadherin-mediated cell-to-cell contact (1). EpCAM is composed of a large extracellular domain (EpEx), a single transmembrane domain and a short 26-amino acid intracellular domain (EpICD) $(1,2)$. EpCAM is frequently expressed in normal epithelial cells, and is reported to be widely upregulated in various cancers and cancer-initiating cells (3-5). The high expression of membranous EpCAM in a variety of cancers analyzed by immunohistochemistry has rendered EpCAM as an ideal immunotherapeutic target $(2,3)$. However, results from clinical trials of EpCAM-specific monoclonal antibodies in various cancers have shown limited efficacy $(6,7)$. The cleavage of EpEx by the protease tumor necrosis factor $\alpha$-converting enzyme (TACE) and its shedding have been shown to result in the release of its intracellular domain EpICD (8). Released EpICD associates with four-anda-half LIM domain protein 2 (FHL2), forms a nuclear complex with components of the Wnt signal pathway ( $\beta$-catenin, Lef-1), and induces gene transcription, resulting in activation of oncogenic signaling (8). This proposed mechanism of regulated intramembranous proteolysis (RIP)-mediated loss of EpCAM from the cancer cell membrane and activation of oncogenic signaling via translocated nuclear EpICD might account for the restricted therapeutic efficacy of the EpCAM-targeted immunotherapy using monoclonal antibodies to target EpEx. Indeed, nuclear expression of EpICD in some types of cancer has been reported (9), and EpICD has been proposed as a new therapeutic target for cancer cells $(9,10)$. However, little is known about the roles of EpICD in carcinogenesis and cancer progression.

$\beta$-catenin plays an important role in both adhesion and signal transduction of epithelial cells, depending on the intracellular localization. $\beta$-catenin forms adherens junctions through conjugation with $\alpha$-catenin and E-cadherin at the plasma membrane. However, $\beta$-catenin can also act as a main regulator of transcription through DNA-binding proteins, such as TCF/LEF family members in the nucleus (11). Previous studies suggest that the activation of the Wnt/ $\beta$-catenin signaling 
pathway plays an important role in human cancer invasion and metastasis (11-14). Nuclear expression of $\beta$-catenin, a hallmark of an active $\beta$-catenin-dependent Wnt pathway, was found predominantly at the invasive front of cancers (12-14).

Extrahepatic cholangiocarcinoma (ECC) arises from cholangiocytes of extrahepatic bile ducts. ECC has a devastating prognosis, and its poor prognosis is associated with extensive local tumor invasion and metastasis $(15,16)$. Despite advances in screening for early detection and in therapy over recent decades, the overall survival rates for patients with ECC have not been improved significantly (17). Thus, to improve the prognosis of ECC patients, identification of new molecular mechanisms of invasion and metastasis of ECC is essential. Based on the observation of nuclear translocation of EpICD in a complex with $\beta$-catenin and Lef, which are both components of the Wnt signaling pathway, we hypothesized that spatial localization of EpICD and its mutual interaction with $\beta$-catenin may be important to ECC cancer cell invasion and metastasis.

In the present study, we examined: i) the localization and expression of EpEx, EpICD and $\beta$-catenin in surgical specimens of ECCs from 79 patients with a focus on the relationship between nuclear expression of EpICD and $\beta$-catenin; ii) the effect of EpICD in cholangiocarcinoma (CC) cell growth and proliferation; iii) the effect of EpICD in EpCAM target gene expression; and iv) the role of EpICD in the migration and invasion of CC cells. We also examined whether EpCAM silencing by small interfering RNA (siRNA) could affect cell growth, migration and invasiveness in CC cells.

\section{Materials and methods}

Materials. This study was approved by the Institutional Review Board (IRB) of Chonbuk National University Hospital. Surgical specimens of 79 formalin-fixed, paraffin-embedded ECCs were obtained from Surgical Pathology Archives of Chonbuk National University Hospital, Korea between 1998 and 2010. Of the 79 patients with ECC, 56 (70.9\%) were men and $23(29.1 \%)$ were women. The tumors were histologically graded as 26 cases $(23.9 \%)$ of well-differentiated, 46 cases $(58.3 \%)$ of moderately-differentiated and seven cases $(8.8 \%)$ of poorly-differentiated ECC.

We used the four human CC cell lines, designated as JCK, Cho-CK, Choi-CK and SCK, which were established in our laboratory (18). In addition, the RBE cell line was purchased from the RIKEN BRC cell bank (Tsukuba, Japan). All CC cell lines were maintained in Dulbecco's modified Eagle's medium (DMEM) supplemented with penicillin/streptomycin and $10 \%$ fetal bovine serum (Gibco BRL, Gaithersburg, MD, USA). The cells were cultured at $37^{\circ} \mathrm{C}$ in a $5 \% \mathrm{CO}_{2}$ humidified incubator.

Immunohistochemical staining. For immunohistochemical staining, we used a polymer intense detection system using the Bond-Max Automatic stainer (Leica, Bannockburn, IL, USA) in accordance with the manufacturer's instructions. Briefly, after deparaffinization, the tissue sections were treated with a microwave antigen retrieval procedure in $0.01 \mathrm{M}$ sodium citrate buffer ( $\mathrm{pH}$ 6.0) for $12 \mathrm{~min}$. The samples were incubated with anti-EpCAM (Calbiochem, La Jolla, CA, USA) for the extracellular domain of EpCAM (EpEX), anti-EpICD for the intracellular domain of EpCAM (C-terminus, cytoplasmic domain of EpCAM; 1144-1) (Epitomics, Burlingame, CA, USA) and anti- $\beta$-catenin (BD Biosciences, San Jose, CA, USA) antibodies for $20 \mathrm{~min}$. Peroxidase activity was detected with the enzyme substrate 3-amino-9-ethyl carbazole. Samples with EpEx, EpICD and $\beta$-catenin staining of $\geq 10 \%$ of the tumor cells with strong intensity were defined as positive.

Plasmid cDNA and small interfering RNA transfection. For EpICD plasmid cDNA transfection, EpICD cDNA (NCBI accession number NM_002354.2; EpCAM cytoplasmic domain) was synthesized from CosmoGenetech Co., Ltd. (Seoul, Korea) and inserted into the XhoI and BamHI sites of pEGFP-N1 vector (Clontech, Palo Alto, CA, USA). Transfection of EpICD plasmid DNA was performed with lipofectamine 2000 transfection reagent (Invitrogen, Carlsbad, CA, USA) following the manufacturer's protocol. At $48 \mathrm{~h}$ after transfection, the cells were collected and used for further experiments.

Small interfering RNA (siRNA) was used for silencing EpCAM expression as previously described (19). EpCAM siRNA and negative control were purchased from Bioneer Corp. (Daejeon, Korea). Sequences for EpCAM-specific siRNA and negative control siRNA were as follows: sense: 5'-GUGAGAACCUACUGGAUCA(dTdT)-3', antisense: 5'-UGAUCCAGUAGGUUCUCAC(dTdT)-3'; and negative control: sense: 5'-CCUACGCCACCAAUUUCGU(dTdT)-3', antisense: 5'-ACGAAAUUGGUGGCGUAGG(dTdT)-3'. Transfection of siRNA was performed with lipofectamine RNAiMAX transfection reagent (Invitrogen) following the manufacturer's protocol.

Western blotting. Western blotting of EpEx (Calbiochem) and EpICD (Epitomics) in CC cell lines was performed as previously described (20). To determine the effect of forced expression of EpICD, we also examined the expression profile of E-cadherin (BD Biosciences), active $\beta$-catenin (Merck Millipore, Billerica, MA, USA), c-Myc (Abcam, Cambridge, UK), and cyclin D1 (Santa Cruz Biotechnology, Santa Cruz, CA, USA) in EpICD-transfected CC cell lines. Blots were developed using secondary antibody, and immune complexes were visualized using an enhanced chemiluminescence detection system (Amersham Biosciences, Buckinghamshire, UK), prior to exposure to a luminescent image analyzer (LAS-3000, Fuji Film, Tokyo, Japan).

Cell growth and proliferation assay. The effects of the EpICD gene transfection and silencing of EpCAM on cell proliferation were assessed by MTT (3-(4,5-dimethylthiazol-2-yl)-2,5-diphenyltetrazolium bromide) (Sigma, St. Louis, MO, USA) and BrdU cell proliferation assay, as described previously (19).

In vitro assay for cellular migration and invasion. Cell migration was assessed in a 24-Transwell migration assay (Corning Life Sciences, Acton, MA, USA) and the cell invasion assay was performed using a 24-Transwell BioCoat Matrigel invasion chamber (BD Biosciences), as previously described (20).

Statistical analysis. Statistical analysis was performed using SPSS version 15.0 (SPSS, Chicago, IL, USA). Statistical 

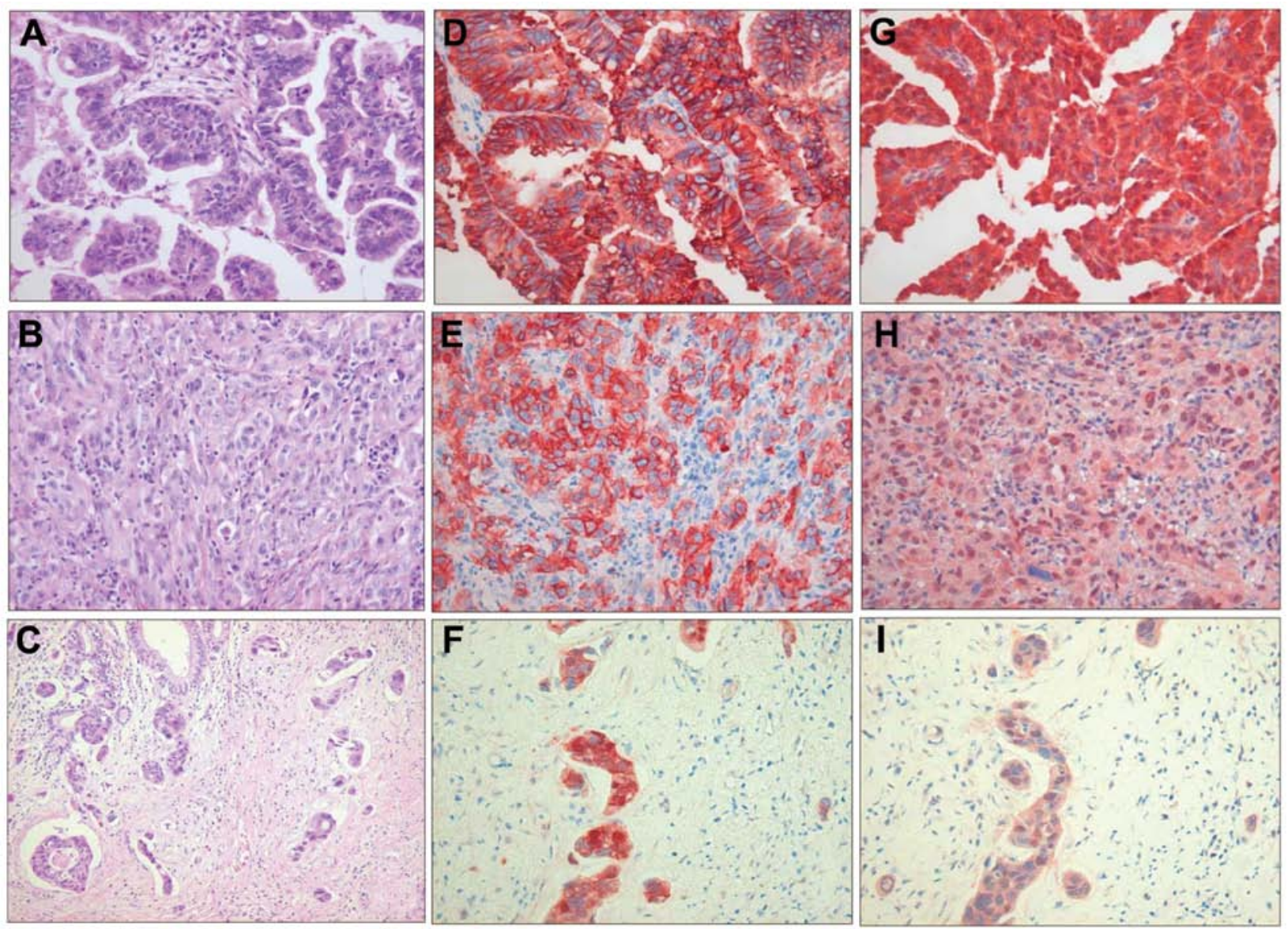

Figure 1. Representative immunostaining for EpEx, EpICD and $\beta$-catenin in extrahepatic cholangiocarcinoma. (A) Well-differentiated cancer (H\&E, x200) (B) Poorly differentiated cancer (H\&E, x200); (C) Moderately differentiated cancer cells at the invasive front (H\&E, x200). (D and E) Cancer cells with selective membrane staining for EpEx in well differentiated cancer and poorly differentiated cancer, respectively (x400). Note the absence of immunoreactivity in the nucleus. $(\mathrm{G}$ and $\mathrm{H})$ Nuclear and cytoplasmic EpICD immunoreactivity of cancer cells in well differentiated cancer and poorly differentiated cancer, respectively (x400). (F and I) Nuclear co-localization of $\beta$-catenin (F) and EpICD (I) in the same cancer cells at the invasive front (x400).

significance was determined by Chi-square test and appropriate Student's t-test. All experiments were repeated a minimum of three times. A P-value of $<0.05$ was considered statistically significant.

\section{Results}

EpEx, EpICD and $\beta$-catenin expression in extrahepatic cholangiocarcinoma. The positive expression of EpEx was observed in $98.7 \%$ (78 of 79) of ECC tissues. In ECC cells, EpEx expression was predominantly localized to the membrane (Fig. 1A, B, D and E). EpEx was not detected in any of the nuclei of tumor cells. Increased nuclear and/ or cytoplasmic expression with reduced or absent membrane expression of EpICD was found in ECC cells (Fig. 1C and F). Nuclear expression of EpICD was observed in $27.8 \%$ (22 of 79 ) of ECC tissues. No nuclear expression of EpICD was present in non-neoplastic bile duct cells. Nuclear expression of $\beta$-catenin was observed in $12.6 \%$ (10 of 79) of ECC tissues. Frequent nuclear co-localization of EpICD and $\beta$-catenin was observed in cancer cells forming the invasive front (Fig. 1G, H and I). Nuclear translocation of EpICD was significantly higher in poorly and moderately differentiated tumor cells compared to well-differentiated tumor cells $(\mathrm{P}=0.021)$ (Table $\mathrm{I})$. There was a significant

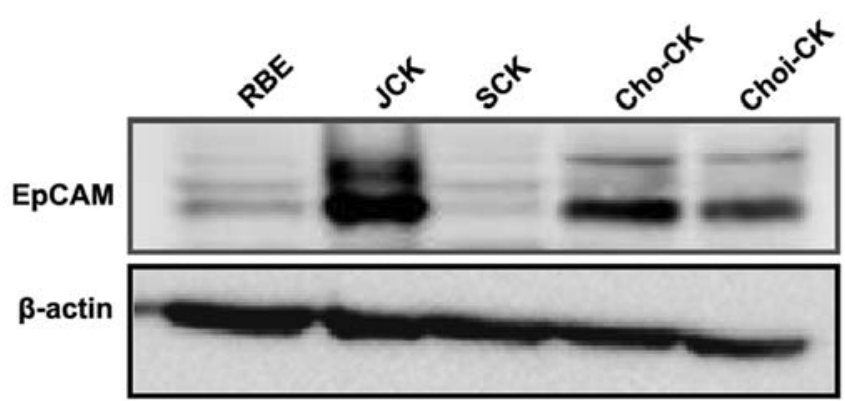

Figure 2. Western blot analysis of EpCAM expression in five different cholangiocarcinoma cell lines. The expression level of EpCAM protein was higher in JCK and Cho-CK cell lines than in RBE and SCK cell lines.

correlation between the nuclear translocation of EpICD and nuclear expression of $\beta$-catenin $(\mathrm{P}=0.015)$ (Table I). Aberrant nuclear expression, and often co-localization, of EpICD and $\beta$-catenin in cancer cells forming the invasive front suggests that they are engaged in local interactions and play important roles in ECC invasion.

EpCAM expression level in cholangiocarcinoma cell lines. The expression level of EpCAM protein was higher in the JCK and Cho-CK cells than in the other cell lines. Choi-CK cells 

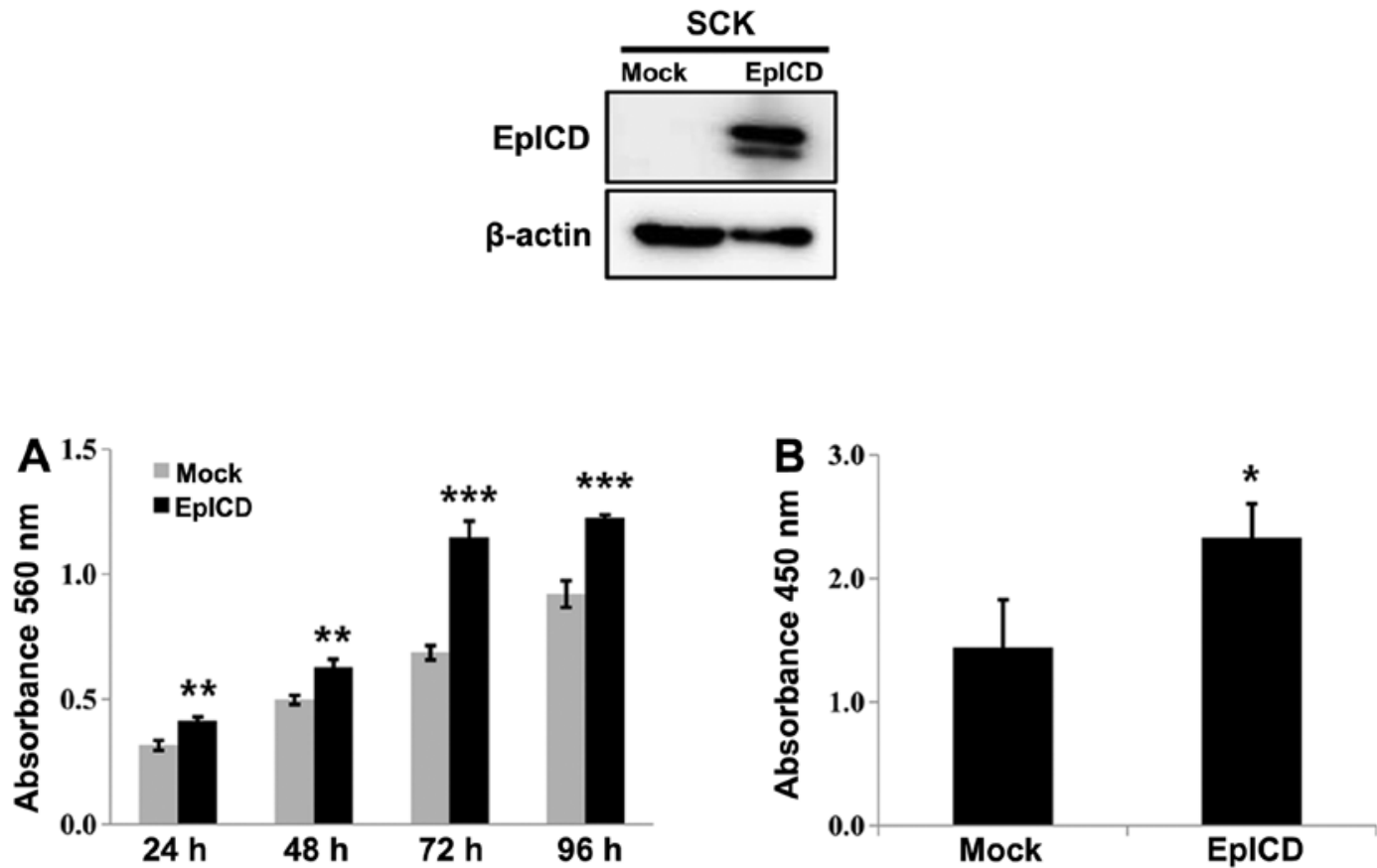

Figure 3. (A) The time-dependent effects of EpICD on cell growth. The EpICD-overexpressing SCK cells revealed significantly increased cell growth when compared to control cells $\left({ }^{* *} \mathrm{P}<0.01 ;{ }^{* * * *} \mathrm{P}<0.001\right)$. (B) In EpICD-overexpressing SCK cells, there was a significant increase in the BrdU incorporation when compared to control cells $($ ( $\mathrm{P}<0.05)$.

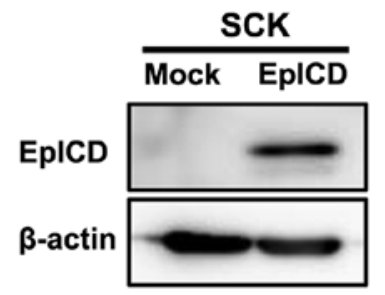

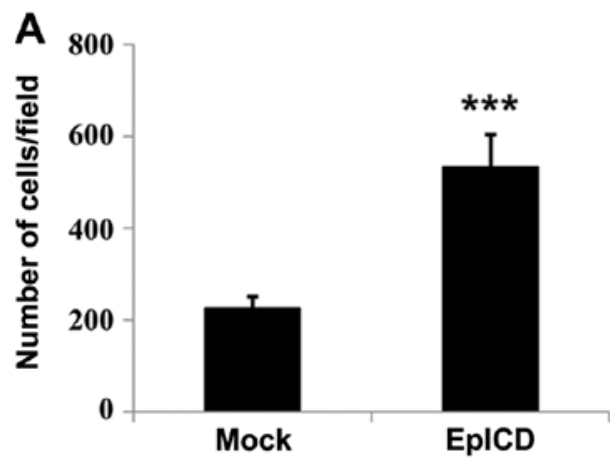

Migration

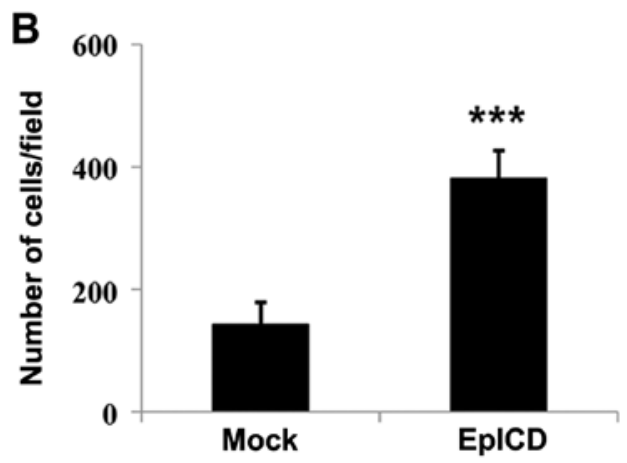

Invasion

Figure 4. In vitro cell migration and invasion assay. (A) For the EpICD-transfected SCK cells, cell migration was increased by 2.4-fold when compared with that of the control $\left({ }^{* * * *} \mathrm{P}<0.001\right)$. (B) The EpICD-transfected SCK cell invasion was increased by 2.7 -fold when compared with that of the control $\left({ }^{* * *} \mathrm{P}<0.001\right)$.

showed low EpCAM protein level that was barely detected in the SCK and RBE cells (Fig. 2).

Effect of EpICD expression on cell growth, proliferation, migration and invasion. SCK cells (minimal EpCAM protein expression) were transiently transfected with EpICD cDNA. The EpICD-overexpressed SCK cells revealed a significant time-dependent increase in cell growth when compared to control cells $(\mathrm{P}<0.01 ; \mathrm{P}<0.001)$ (Fig. 3A). In EpICDoverexpressing SCK cells, there was a significant increase in 


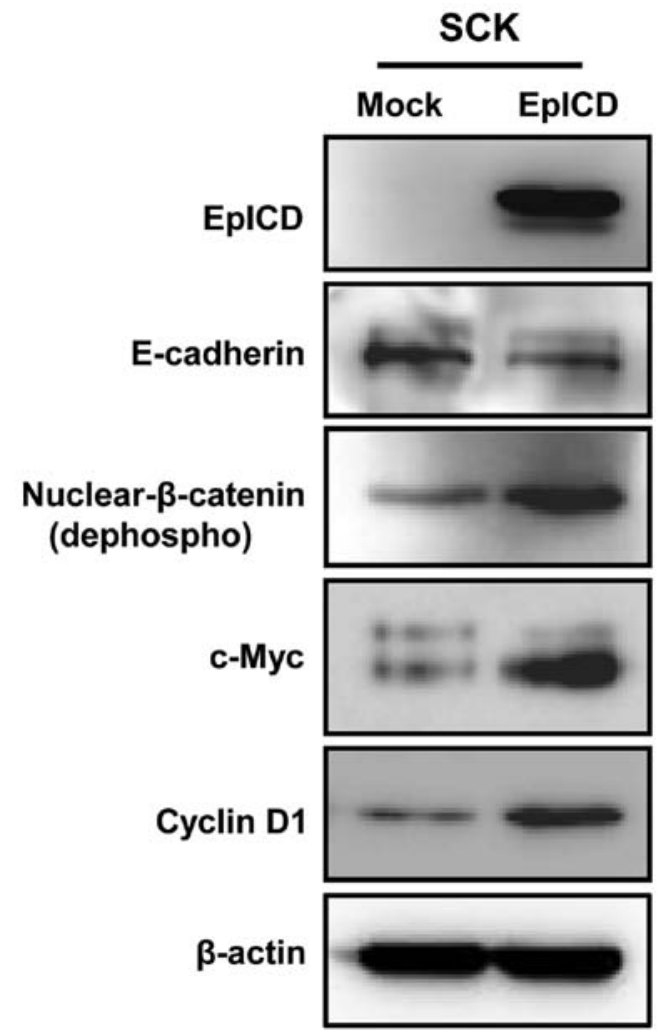

Figure 5. The enhanced expression levels of the active form of the $\beta$-catenin and EpCAM target genes, such as c-myc and cyclin D1, in the EpICDoverexpressing SCK cells.

the BrdU incorporation when compared to that of control cells $(\mathrm{P}<0.05)$ (Fig. 3B). Overexpression of EpICD in SCK cells leads to significantly increased cell migration and invasion when compared to control cells ( $\mathrm{P}<0.001)$ (Fig. 4A and B).

Overexpression of EpICD induces expression of EpCAM target genes and nuclear $\beta$-catenin. The expression levels of EpICD and the active form $\beta$-catenin (nuclear form) were significantly increased by 11- and 2.3-fold, respectively, in the EpICD-transfected SCK cells. Also, the expression of representative EpCAM target genes, such as $c$-myc and cyclin D1, were increased by 3.1- and 2.5-fold, respectively. However, overexpression of EpICD in the SCK cells decreased the expression level of E-cadherin by 1.3-fold (Fig. 5).

Silencing EpCAM decreases cell growth and proliferation. Cho-CK and JCK cells (high EpCAM protein expression) were transiently transfected with EpCAM-specific siRNA as well as scrambled siRNA. Transfection with EpCAM siRNA resulted in a marked decrease in the expression of EpCAM at $48 \mathrm{~h}$ post-transfection in Cho-CK cells (Fig. 6A). Silencing EpCAM gene expression in Cho-CK cells by EpCAM siRNA resulted in significant inhibition of cell growth when compared to that of the control $(\mathrm{P}<0.05)$ (Fig. 6B). In EpCAM siRNAtransfected Cho-CK cells, there was a significant decrease in the BrdU incorporation when compared to that of control $(\mathrm{P}<0.05)$ (Fig. 6C). Silencing EpCAM gene expression in JCK cells by EpCAM siRNA also decreased the cell growth and BrdU incorporation compared to those of the control, but the difference was not statistically significant.
Table I. Association between nuclear expression of $\beta$-catenin, histologic tumor grade and nuclear translocation of EpICD in extrahepatic cholangiocarcinoma.

\begin{tabular}{lccc}
\hline & $\begin{array}{c}\text { EpICD } \\
\text { nuclear } \\
\text { translocation }\end{array}$ & $\begin{array}{c}\text { EpICD } \\
\text { no } \\
\text { translocation }\end{array}$ & P-value \\
\hline Tumor grade & 5 & 21 & $<0.05$ \\
Well & 12 & 34 & \\
Moderately & 5 & 2 & \\
Poorly & & & $<0.05$ \\
$\begin{array}{l}\beta \text {-catenin } \\
\text { expression }\end{array}$ & & & \\
Nuclear translocation & 6 & 4 & \\
No translocation & 16 & 53 & \\
\hline
\end{tabular}

Silencing EpCAM suppresses cell migration and invasion. Silencing EpCAM gene expression in Cho-CK and JCK cells significantly inhibited migratory ability when compared to control ( $\mathrm{P}<0.001$ and $\mathrm{P}<0.05$, respectively). Silencing EpCAM gene expression in Cho-CK and JCK cells also significantly inhibited the invasiveness when compared to that of the control ( $\mathrm{P}<0.01$ and $\mathrm{P}<0.05$, respectively) (Fig. 7).

\section{Discussion}

EpCAM is highly upregulated in most human epithelial cancers, and several biologic roles of EpCAM in carcinomas have been reported (2-5). EpCAM is primarily a cell adhesion molecule that inhibits cell scattering, thereby preventing tumor cell invasion and metastasis $(1,21)$. Many studies indicate that the role of EpCAM is not limited to cell adhesion; it has been shown to be widely involved in cell proliferation, migration, invasion and cell signaling $(4,5,8,10,22,23)$. It has been proposed that formation of EpICD complex with the scaffolding protein FHL2 plus Lef1 and $\beta$-catenin accounts for activation of the oncogenic potential of EpCAM $(4,8,10)$. Nuclear localization of EpICD was first reported in colon cancer cells (8); subsequently, its nuclear expression has been frequently observed in various cancers (9). Since the roles of EpCAM on the biological behavior of cancer cells depend on the cancer phenotype, we investigated the significance of nuclear EpICD and its potential relationship with $\beta$-catenin in ECC.

This study was the first to demonstrate the following findings in regards to EpICD expression in ECC: i) nuclear expression of EpICD and $\beta$-catenin was detected in $27.8 \%$ and $12.6 \%$ of the 79 ECC patients, respectively, ii) a significant correlation was observed between the nuclear expression of EpICD and $\beta$-catenin in ECC cells with frequent co-localization of EpICD and $\beta$-catenin in cancer cells forming the invasive front, iii) nuclear expression of EpICD was significantly associated with high tumor grade, iv) forced overexpression of EpICD in CC cells increased the expression levels of the active form of $\beta$-catenin and EpCAM target genes, such as c-myc and cyclin D1, v) the overexpression of EpICD significantly increased cell growth and proliferation 
A Cho-CK

EpCAM

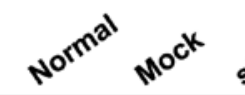<smiles>C1CC2CCC(C1)O2</smiles>

$\beta$-actin
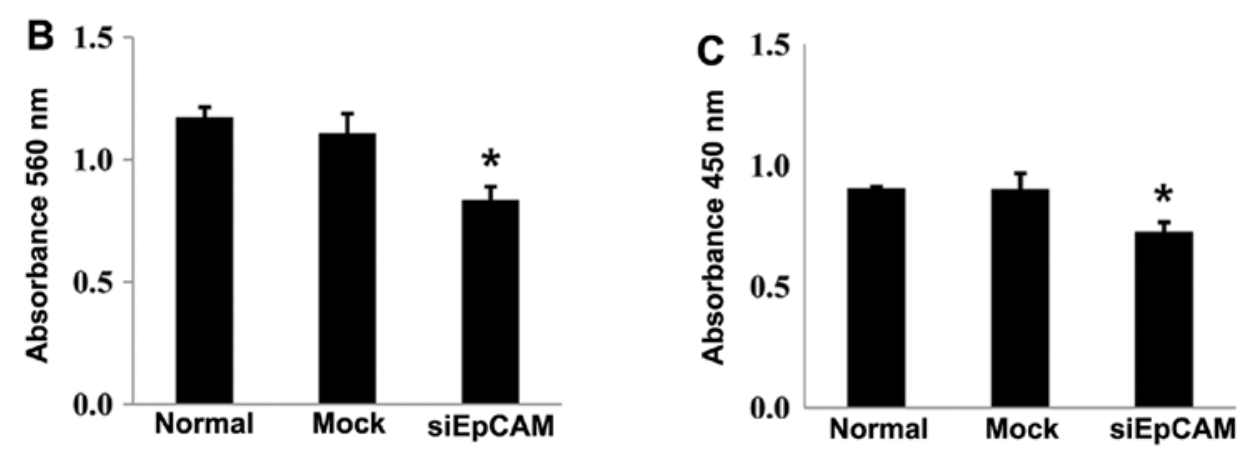

Figure 6. XTT and Brdu proliferation assay in EpCAM downregulated Cho-CK cell line. (A) Transfection with EpCAM siRNA effectively decreased the expression of EpCAM in Cho-CK cells. (B) The XTT assay demonstrated a significant decrease in cell growth compared to that of the control ("P<0.05). (C) In the BrdU assay, EpCAM downregulated Cho-CK cells had significantly decreased cell proliferation compared to control ("P<0.05).
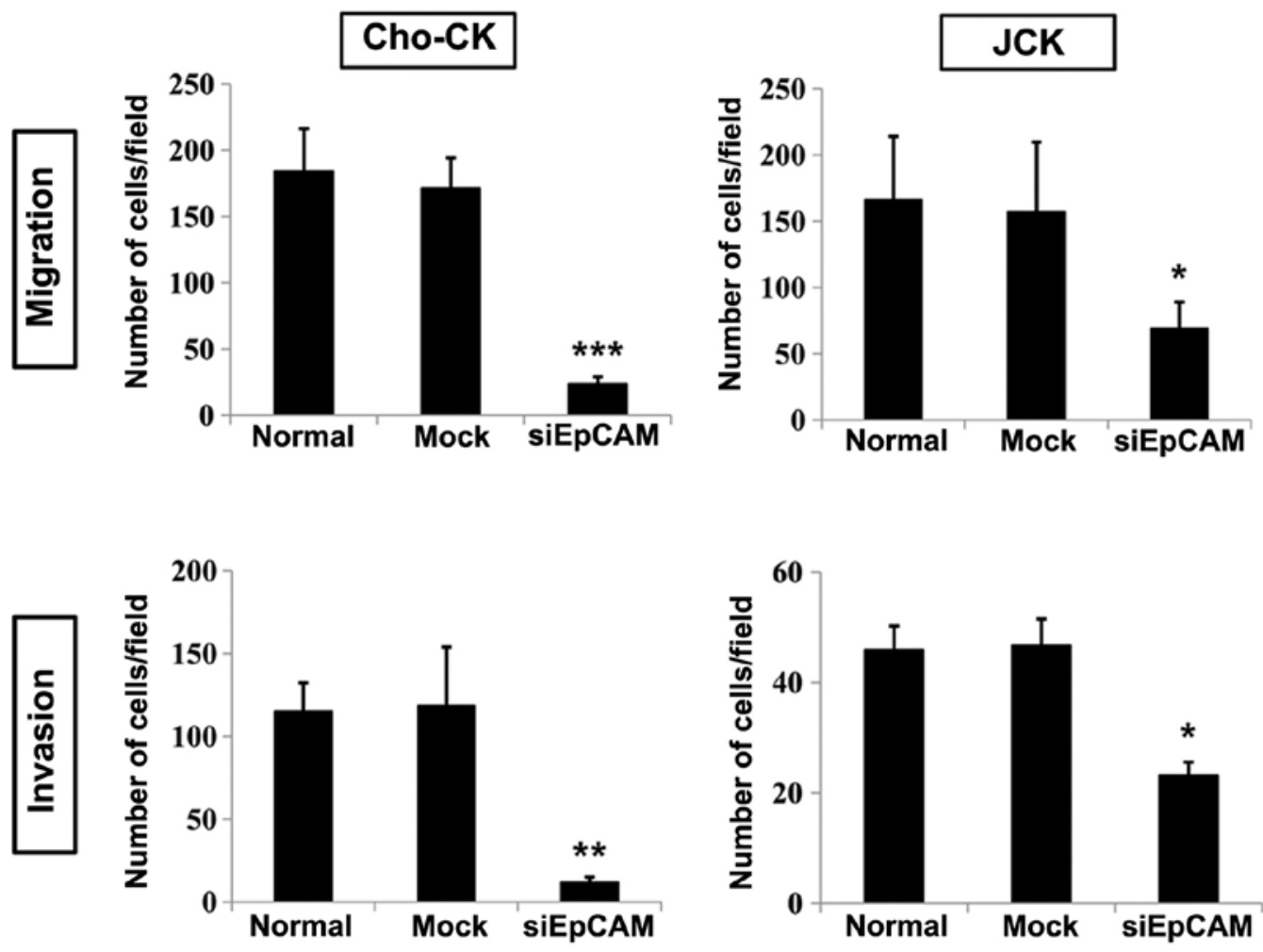

Figure 7. In vitro cell migration and invasion assay in EpCAM downregulated cholangiocarcinoma cell lines. Cell migration and invasion of EpCAM silencing in Cho-CK cells were decreased by 7 - and 10 -fold, respectively, over the control $\left({ }^{* *} \mathrm{P}<0.01 ;{ }^{* * * *} \mathrm{P}<0.001\right)$. For the EpCAM silencing in JCK cells, cell migration and invasion were decreased by 2.3 - and 2 -fold, respectively, when compared with those of the control (" $\mathrm{P}<0.05)$.

in CC cells, and vi) the overexpression of EpICD enhanced the cell motility and invasiveness of CC cells. Furthermore, inhibition of the EpCAM expression in the EpCAM highexpressing Cho-CK and JCK cells by siRNA significantly 
decreased cell proliferation, migration and invasion. These findings clearly indicate the important role of nuclear co-expression of EpICD and $\beta$-catenin and their local interactions in ECC progression and invasion.

The morbidity and mortality of ECC patients predominantly depends on the degree of invasion and metastasis (15-17). To investigate whether nuclear EpICD is involved in ECC cell invasion in cooperation with $\beta$-catenin, we performed immunohistochemistry using antibodies to EpICD and $\beta$-catenin in ECC specimens. In this study, we found that budding ECC cells of the invasive margin displayed increased translocation of EpICD into the nucleus, which was frequently concurrent with nuclear $\beta$-catenin expression. Notably, nuclear EpICD staining of ECC specimens correlated significantly with nuclear expression of $\beta$-catenin and de-differentiation of tumors. We also found that the forced overexpression of EpICD in ECC cells increased expression levels of the active form of $\beta$-catenin (nuclear $\beta$-catenin) and enhanced the cell motility and invasiveness of CC cells. Furthermore, consistent with the promoting role of EpICD in cell invasion, knockdown of EpCAM by siRNA dramatically decreases cell migration and invasiveness of ECC cells. These findings are in agreement with results from a previous study demonstrating that the oncogenic potential of EpCAM is activated by release of the EpICD into the cytoplasm, which can signal into the nucleus by engagement of elements of the Wnt pathway $(4,8)$.

Evidence for the important role of EpICD in cancer progression and invasion is increasing (25-28). Concomitant nuclear expression of EpICD and $\beta$-catenin in anaplastic and aggressive thyroid cancers has been reported and nuclear EpICD expression has been shown to be correlated with poor overall survival of thyroid cancer patients (24). Expression of EpCAM signaling pathway members including EpICD and $\beta$-catenin in gastric carcinoma also has been previously described (25). Fong et al have shown that loss of membranous EpICD is frequently observed in pancreatic cancer and can predict poor prognosis of pancreatic cancer patients (26), and the expression level of EpICD in colon cancer is associated with tumor grade (27). Similar results have been reported by Gosens et al, who described a focal loss of membranous EpEx at the invasive margin in colorectal cancer (28). Based on the above observations, our findings of nuclear translocation of EpICD in parallel with aberrant nuclear expression of $\beta$-catenin in cancer cells forming the invasive front and increased expression of nuclear $\beta$-catenin by EpICD suggest that activation of EpICD signaling can induce Wnt signaling, thus accounting for the ECC de-differentiation and increased invasive potential of tumor cells. However, how EpICD promotes tumor cell invasion remains elusive. Matrix metalloproteinases (MMPs) are a major group of proteolytic enzymes that are implicated in migration and invasion of cancer cells (29). Recently, Denzel et al reported that MMP7 is a novel target gene of EpCAM signaling, and transcription of the $m m p 7$ gene is dependent on nuclear translocation of EpICD and Wnt signaling through $\beta$-catenin and Lef-1 consensus sites (30).

This study showed that overexpression of EpICD resulted in a significant increase in the rate of cell proliferation of CC cells. We also demonstrated that overexpression of EpICD upregulated the expression of proto-oncogene $c$-myc and the cell cycle regulating gene $c y c l i n D 1$. These findings are in agreement with those from a previous study showing that EpICD regulates the activity of reprogramming genes, including $c$-myc (27). Litvinov et al first showed that EpCAM expression was correlated with the proliferative activity of tumor cells as demonstrated by Ki-67 expression in cervical intraepithelial neoplasia (31). Accumulating evidence suggests that EpCAM has a critical role in the generation of proliferative signals into the nucleus via RIP to release EpICD $(4,8-10,27,30,32)$. Expression of EpICD in the absence of EpCAM is sufficient to induce proliferation signals both in vitro and in vivo (8). EpICD alone induces a significant increase in cell proliferation, whereas EpCAM silencing results in a significant decrease in cell number, which is reversed upon EpICD co-transfection in hypopharynx carcinoma cells (32). Our findings, together with those from previous studies, suggest that nuclear translocation of EpICD is necessary and sufficient for cell cycle progression in ECC. This notion is supported by the fact that the ability to rapidly upregulate the proto-oncogene c-myc as well as cyclin A and E can be mediated solely by EpICD $(8,27,35)$. Additionally, it has been shown that EpICD upregulates the expression of cyclin D1 at the transcriptional level (34). In addition, we found that EpCAM gene silencing by siRNA dramatically reduced $\mathrm{CC}$ cell proliferation capacity. This is confirmation of earlier findings that EpCAM blockage with siRNA inhibits cell proliferation and tumorigenicity in various cancers $(19,32-34)$.

In conclusion, our data indicate that EpICD is translocated into the nucleus in a proportion of ECCs and significantly correlates with nuclear expression of $\beta$-catenin, especially in cancer cells forming the invasive front. The overexpression of EpICD in the CC cells significantly enhanced cell proliferation, migration and invasiveness with a concurrent increase in the expression of nuclear $\beta$-catenin and EpCAM target genes. Moreover, silencing of EpCAM gene expression significantly inhibits tumor cell proliferation and decreases the migration and invasiveness of CC cells. These findings strongly suggest the importance of nuclear co-expression of EpICD and $\beta$-catenin and their mutual interactions in ECC progression and invasion. EpCAM has been targeted in clinical trials using monoclonal antibodies directed against EpEx in various cancers $(2,3,6,7)$. We believe that the EpICD-mediated EpCAM nuclear signaling pathway is the main contributor to the oncogenic signaling pathway. Nuclear expression of EpICD and its role in oncogenic signaling, cancer cell proliferation and invasiveness provide a strong basis for further investigation of anti-EpICD-targeted therapy.

\section{Acknowledgements}

This study was supported by the National Research Foundation of Korea (NRF) grant funded by the Korean Government (MSIP) (no. 2008-0062279).

\section{References}

1. Litvinov SV, Balzar M, Winter MJ, et al: Epithelial cell adhesion molecule (Ep-CAM) modulates cell-cell interactions mediated by classic cadherins. J Cell Biol 139: 1337-1348, 1997.

2. Baeuerle PA and Gires O: EpCAM (CD326) finding its role in cancer. Br J Cancer 96: 417-423, 2007.

3. Went PT, Lugli A, Meier S, et al: Frequent EpCam protein expression in human carcinomas. Hum Pathol 35: 122-128, 2004. 
4. Munz M, Baeuerle PA and Gires O: The emerging role of EpCAM in cancer and stem cell signaling. Cancer Res 69: 5627-5629, 2009.

5. Patriarca C, Macchi RM, Marschner AK and Mellstedt $\mathrm{H}$ : Epithelial cell adhesion molecule expression (CD326) in cancer: a short review. Cancer Treat Rev 38: 68-75, 2012.

6. Schmidt M, Scheulen ME, Dittrich C, et al: An open-label, randomized phase II study of adecatumumab, a fully human antiEpCAM antibody, as monotherapy in patients with metastatic breast cancer. Ann Oncol 21: 275-282, 2010.

7. Niedzwiecki D, Bertagnolli MM, Warren RS, et al: Documenting the natural history of patients with resected stage II adenocarcinoma of the colon after random assignment to adjuvant treatment with edrecolomab or observation: results from CALGB 9581. J Clin Oncol 29: 3146-3152, 2011.

8. Maetzel D, Denzel S, Mack B, et al: Nuclear signalling by tumour-associated antigen EpCAM. Nat Cell Biol 11: 162-171, 2009.

9. Ralhan R, He HC, So AK, et al: Nuclear and cytoplasmic accumulation of Ep-ICD is frequently detected in human epithelial cancers. PLoS One 5: e14130, 2010.

10. Carpenter G and Red Brewer M: EpCAM: another surface-tonucleus missile. Cancer Cell 15: 165-166, 2009.

11. Schmalhofer O, Brabletz S and Brabletz T: E-cadherin, betacatenin, and ZEB1 in malignant progression of cancer. Cancer Metastasis Rev 28: 151-166, 2009.

12. Polette M, Mestdagt M, Bindels S, et al: Beta-catenin and ZO-1: shuttle molecules involved in tumor invasion-associated epithelial-mesenchymal transition processes. Cells Tissues Organs 185: 61-65, 2007.

13. Vincan E and Barker N: The upstream components of the Wnt signalling pathway in the dynamic EMT and MET associated with colorectal cancer progression. Clin Exp Metastasis 25: 657-663, 2008

14. Paul S and Dey A: Wnt signaling and cancer development: therapeutic implication. Neoplasma 55: 165-176, 2008.

15. Khan SA, Thomas HC, Davidson BR and Taylor-Robinson SD Cholangiocarcinoma. Lancet 366: 1303-1314, 2005.

16. Blechacz B and Gores GJ: Cholangiocarcinoma: advances in pathogenesis, diagnosis, and treatment. Hepatology 48: 308-321, 2008.

17. van der Gaag NA, Kloek JJ, de Bakker JK, et al: Survival analysis and prognostic nomogram for patients undergoing resection of extrahepatic cholangiocarcinoma. Ann Oncol 23: 2642-2649, 2012.

18. Kim DG, Park SY, You KR, et al: Establishment and characterization of chromosomal aberrations in human cholangiocarcinoma cell lines by cross-species color banding. Genes Chromosomes Cancer 30: 48-56, 2001.

19. Bae JS, Noh SJ, Jang KY, et al: Expression and role of epithelial cell adhesion molecule in dysplastic nodule and hepatocellular carcinoma. Int J Oncol 41: 2150-2158, 2012.

20. Kwon CY, Kim KR, Choi HN, et al: The role of serum response factor in hepatocellular carcinoma: Implications for disease progression. Int J Oncol 37: 837-844, 2010.
21. Litvinov SV, Velders MP, Bakker HA, Fleuren GJ and Warnaar SO: Ep-CAM: a human epithelial antigen is a homophilic cell-cell adhesion molecule. J Cell Biol 125: 437-446, 1994.

22. van der Gun BT, Melchers LJ, Ruiters MH, de Leij LF, McLaughlin PM and Rots MG: EpCAM in carcinogenesis: the good, the bad or the ugly. Carcinogenesis 31: 1913-1921, 2010.

23. Trzpis M, McLaughlin PM, de Leij LM and Harmsen MC Epithelial cell adhesion molecule: more than a carcinoma marker and adhesion molecule. Am J Pathol 171: 386-395, 2007.

24. Ralhan R, Cao J, Lim T, Macmillan C, Freeman JL and Walfish PG: EpCAM nuclear localization identifies aggressive thyroid cancer and is a marker for poor prognosis. BMC Cancer 10: 331,2010

25. Warneke VS, Behrens HM, Haag J, et al: Members of the EpCAM signalling pathway are expressed in gastric cancer tissue and are correlated with patient prognosis. Br J Cancer 109: 2217-2227, 2013.

26. Fong D, Moser P, Kasal A, et al: Loss of membranous expression of the intracellular domain of EpCAM is a frequent event and predicts poor survival in patients with pancreatic cancer. Histopathology 64: 683-692, 2014.

27. Lin CW, Liao MY, Lin WW, Wang YP, Lu TY and Wu HC: Epithelial cell adhesion molecule regulates tumor initiation and tumorigenesis via activating reprogramming factors and epithelial-mesenchymal transition gene expression in colon cancer. J Biol Chem 287: 39449-39459, 2012.

28. Gosens MJ, van Kempen LC, van de Velde CJ, van Krieken JH and Nagtegaal ID: Loss of membranous Ep-CAM in budding colorectal carcinoma cells. Mod Pathol 20: 221-232, 2007.

29. Kessenbrock K, Plaks V and Werb Z: Matrix metalloproteinases: regulators of the tumor microenvironment. Cell 141: 52-67, 2010.

30. Denzel S, Mack B, Eggert C, et al: MMP7 is a target of the tumour-associated antigen EpCAM. Int J Exp Pathol 93: 341-353, 2012.

31. Litvinov SV, van Driel W, van Rhijn CM, et al: Expression of Ep-CAM in cervical squamous epithelia correlates with an increased proliferation and the disappearance of markers for terminal differentiation. Am J Pathol 148: 865-875, 1996.

32. Chaves-Pérez A, Mack B, Maetzel D, et al: EpCAM regulates cell cycle progression via control of cyclin D1 expression. Oncogene 32: 641-650, 2013

33. Osta WA, Chen Y, Mikhitarian K, et al: EpCAM is overexpressed in breast cancer and is a potential target for breast cancer gene therapy. Cancer Res 64: 5818-5824, 2004.

34. Yamashita T, Ji J, Budhu A, et al: EpCAM-positive hepatocellular carcinoma cells are tumor-initiating cells with stem/progenitor cell features. Gastroenterology 136: 1012-1024, 2009.

35. Münz M, Kieu C, Mack B, Schmitt B, Zeidler R and Gires O: The carcinoma-associated antigen EpCAM upregulates c-myc and induces cell proliferation. Oncogene 23: 5748-5758, 2004. 